\title{
Post operative Mycobacterium fortuitum infection following laparoscopic inguinal hernia repair: A case report
}

\author{
Kusum Sharma ${ }^{1}$, Suma Appannanavar ${ }^{1}$, Shiv Kumar ${ }^{1}$, Aman Sharma², Meera Sharma ${ }^{1}$ \\ Department of Medical Microbiologyl and Internal Medicine ${ }^{2}$ \\ Post Graduate Institute of Medical Education and Research Chandigarh.
}

\begin{abstract}
Rapidly growing mycobacteria (RGM) are ubiquitous in nature and are associated with opportunistic nosocomial infections requiring prolonged and multiple antibiotics. Here we report a PCR confirmed case of M. fortuitum complex infection in a post laproscopic inguinal hernia repair using prolene mesh. To the best of our knowledge we have for the first time evaluated the role of PCR using specific primers for the diagnosis of $M$. fortuitum complex both from clinical samples and from culture isolate. A 62 year old physician developed low grade fever and pain over the right iliac fossa after an inguinal hernia repair. Mycobacteriological investigation revealed M. fortuitum infection. Subsequently using specific primer, $M$. fortuitum complex DNA was demonstrated both in clinical sample and culture isolate. Patient was started with clarithromycin, doxycycline and streptomycin. Thus, PCR is a promising tool for the rapid diagnosis of difficult to treat infections caused by $M$. fortuitum complex.
\end{abstract}

Key words: Inguinal hernia. Mycobacterium fortuitum, PCR, Post laparoscopic

\section{INTRODUCTION}

Rapidly growing mycobacteria (RGM) are ubiquitous in nature and are associated with opportunistic nosocomial infections. Most of these infections are postoperative and are frequently seen after using devices or implants. ${ }^{1}$ Although infections due to RGM are rare but generally lead to delay in wound healing requiring prolonged and multiple antibiotic therapies. ${ }^{2}$ Among the members of RGM, M. fortuitum are being increasingly reported in various post operative cases. Here we report a PCR confirmed case of $M$. fortuitum complex in a post laproscopic inguinal hernia repair using prolene mesh. To the best of our knowledge we have first time evaluated the role of PCR using specific primers for the diagnosis of $M$. fortuitum complex both from clinical samples and from culture isolate. PCR product was further confirmed as $M$. fortuitum complex by sequencing.

\section{CASE REPORT}

A 62 year old physician underwent laparoscopic right inguinal hernia repair using prolene mesh in a private

\section{Corresponding Author :}

Dr Kusum Sharma MD, Department of Medical Microbiology

Post Graduate Institute of Medical Education and

Research Chandigarh.

Email: sharmakusum9@yahoo.co.in hospital in March 2010. Two weeks after the operative procedure he developed low grade fever and mild pain over the right iliac fossa and patient was given multiple courses of antibiotics for over a period of three weeks. Despite the antibiotic therapy there was no improvement in patient's condition and was referred to our tertiary care centre. At the onset all the antibiotics were stopped and patient was advised ultrasonography which revealed collection of pus in the right inguinal region. In the month of May i.e. 6-8 weeks after the surgery ultrasound guided fine needle aspirations were done on two different occasions. The pus aspirate along with blood sample was sent for routine bacteriological investigation. Both samples were subjected to routine bacteriological culture and sensitivity using standard procedures and were found to be negative. Subsequently in the third week of May repeat ultrasound examinations revealed perimesh collection and around $15-20 \mathrm{ml}$ of pus was aspirated. The aspirate was sent for mycobacterial culture and smear examination and the sample was inoculated onto two Lowenstein Jensen slants (LJ) and BACTEC MGIT 960 system. Part of the sample was subjected for polymerase chain reaction using Mycobacterium tuberculosis complex (MTC) specific gene (IS6110). Microscopy of the Ziehl Neelsen stained smear revealed plenty of polymorphonuclear cells with no organism. IS6110 PCR was also negative for MTC specific gene. Fourteen days after incubation both LJ and BACTEC grew nonchromogenic atypical mycobacterium as was confirmed 
by acid fast staining. Subsequently standard biochemical tests were carried out along with further subculture on two LJ slants and Mac Conkey medium. The organism was found to be a rapid grower (growth within 7 days of subculture) and formed tiny pink colonies on Mac Conkey agar. The rapid growers include $M$ fortuitum, $M$ chelonae, M. abscessus or M. smegmatis. Among the rapid growers the ability to grow on Mac Conkey ruled out $M$. smegmatis and ability to reduce nitrate ruled out M. chelonae and M. abscessus. Thus the isolate was presumptively identified as $M$. fortuitum, for further confirmation the isolate was subjected for PCR targeting M. fortuitum complex specific SOD gene and yielded a PCR product of $275 \mathrm{bp.}^{3}$ (Fig 1). Retrospectively we subjected the pus sample for PCR using the same target and the sample also was positive for $M$. fortuitum complex DNA. The PCR product both from culture which grew atypical mycobacteria as well as from pus sample was confirmed as $M$. fortuitum complex by sequencing. Patient was started with clarithromycin, doxycycline and streptomycin for a period of 18 months. During the follow up period patient developed non healing sinus formation and underwent surgical intervention to remove the mesh.

\section{DISCUSSION}

Mycobacterium fortuitum is a rapidly growing atypical mycobacteria found ubiquitously in the environment. It is frequently isolated from postoperative nosocomial infections in patients who have undergone surgery using various devices and implants like orthopedic prosthesis, peritoneal dialysis catheters, heart valves, pacemaker and mesh for hernia repair. ${ }^{1}$ The case discussed here is patient of M. fortuitum infection following laparoscopic inguinal hernia repair using prolene mesh. There are only few case reports of infections caused by $M$. fortuitum and $M$. chelonae in patients who have undergone laparoscopic surgery from Indian subcontinent. However, all the reports are based on conventional methods like AFB staining and culture. ${ }^{4,5}$ The case reported here is unique due to use of molecular techniques like polymerase chain reaction for the diagnosis of $M$. fortuitum infection.

Infections due to atypical mycobcateria in general and M. fortuitum in particular are known to have chronic course requiring multiple course of antibiotics. Routine bacteriological culture and sensitivity techniques used for the diagnosis may miss these atypical mycobacteria and such patients must be investigated specially for mycobaterial infections. AFB smear examination has its own limitation of lower sensitivity and specificity. Culture isolation and identification of isolate using biochemical reaction are although specific but are time consuming and cumbersome. Nucleic acid amplification techniques can play an important role in the rapid and definitive diagnosis of particular infections as antimicrobial therapy can be instituted as early as possible.

In the case reported here, we presumptively identified the pus isolate as $M$. fortuitum using conventional technique which was further confirmed by PCR with primers specific for $M$. fortuitum complex. In our case, M. fortuitum complex specific DNA was positive also in the clinical sample. With the use of PCR directly on clinical samples the results can be available within 24-48 hours. Patient had received treatment with multiple courses of antibiotics prior to visiting our referral centre. Once the etiological agent was confirmed as M. fortuitum complex, patient was put on appropriate treatment. However, patient developed sinus formation due to the lack of suspicion during the initial presentation and delay in institution of appropriate therapy.

The most probable cause of infection in such patients could be due to break in the operative procedure. Celdran et al have reported two cases of $M$. fortuitum infection following laparoscopic inguinal hernia repair using synthetic mesh. And the probable case of infection in these patients was use of resterilized polypropylene mesh. ${ }^{6}$ In another study from India, Vijayraghavan et al showed that the rinsing water used for cleaning laparoscopes was the source of infection in an outbreak of atypical mycobacteria following laparoscopic surgery. ${ }^{7}$ However, in our case we could not trace the source as the surgery was done in a private hospital.

Diagnosis of infections caused by this atypical mycobacterium is often delayed due to lack of familiarity with the organisms. We want to emphasize that clinicians should be aware of these rare infections which mimic chronic bacterial infections especially in the setting of surgical procedures using devices or scopes and detailed attention to disinfection should be given. These patients should be investigated for mycobacterial infections in general and atypical mycobacterium in particular. A rapid and sensitive technique like PCR is a promising tool for early diagnosis of such infections as specific treatment is essential in the early phase of infections for better outcome. 


\section{ACKNOWLEDGEMENTS}

We extend our gratitude to Dr A Chakrabarti for providing the sequence report of our isolate.

\section{REFERENCES}

1. Verghese S, Madhusudhan B, Senthil MS, Thabitha C, Leelavathy $\mathrm{S}$, Padmaja P, et al. Chronic postoperative wound infection caused by Myocobacterium fortuitum complex. J Commun Dis. 2007; 39(4):257-9.

2. De Groote MA, Huiitt G. infection due to rapidly growing mycobacteria. Clin infect Dis. 2006; 42(2):1756.

3. Kee SJ, Kim SM, Kim SH, Shin MG, Shin JH, Suh SP, et al. Multiplex PCR Assay for Identification of Mycobacterial. Chonnam Med J. 2009; 45:19-26.
4. Gayathri Devi DR, Sridaran D, Indumati VA, Babu PRS, Belwady SMR, Swamy ACV. Isolation of Mycobacterium chelonae from wound infection following Laparscopy: A case report. Ind J Tuber. 2004; 51:149-51.

5. Verghese S, Mullaseri A, Padmaja P, Subhadra AC, Cherian KM. Pacemaker implant site infection caused by atypical mycobacteria. Indian Heart J. 1998; 50(2):201-2.

6. Celdran A, Esteban J, Manas J, Granizo JJ. Wound infections due to Mycobacterium fortuitum after polypropylene mesh inguinal hernia repair. J Hosp Infect. 2007; 66(4):374-7.

7. Vijayaraghavan R, Chandrashekhar R, Sujatha Y, Belagavi CS. Hospital outbreak of atypical mycobacterial infection of port sites after laparoscopic surgery. J Hosp Infect. 2006; 64(4):344-7. 


\section{PDF created with pdfFactory Pro trial version www.pdffactory.com}

